\title{
Fixed frequency Finite Control Set Model Predictive Control For Three-level APF
}

\author{
MaiChao Sha ${ }^{1}$, RuiJin $\mathrm{Zhu}^{1,}{ }^{*}$, XueJiao Gong ${ }^{1}$ \\ ${ }^{1}$ Electric Engineering College, Tibet Agriculture \& Animal Husbandry University 860000, China
}

\begin{abstract}
Active power filter can compensate harmonic and reactive power, Three level neutral point clamp (ANPC) has the characteristics of low output harmonic and low device loss. The control of threelevel active power filter needs to consider multiple objectives. Finite control set model predictive control (FCS-MPC) is a new method which can add constraints and multi-objective control. According to the limit of the switch state, it can track the reference current, which has the characteristics of fast dynamic response and good compensation effect. This method not only keeps the neutral point voltage balance, but also solves the problem of unstable switching frequency.
\end{abstract}

\section{Introduction}

The use of power electronics and the integration of new energy will cause a lot of harmonic pollution and reduce the quality of power grid. In order to improve the power quality and safe use of electricity, many scholars have proposed many solutions to the problem of harmonic suppression, such as starting from the harmonic source, improving the topology structure, reducing the occurrence of harmonics, improving the control method, improving the control accuracy, using multi-level structure and so on APF is the most widely used harmonic control device. Its working principle is to inject the detected harmonic current into the power grid in the opposite direction. APF can not only reduce the harmonic content, but also compensate the reactive power. It can effectively improve the power quality and improve the security of the distribution network.

The harmonic frequencies that APF can manage are widely distributed, so they are used in various occasions. Many algorithms are also proposed for APF control, and the stability and reliability of its application have been further improved. The three-level APF, because of its special structure, can output more vector combinations, and because it is also a harmonic source, its special structure makes its impact on the power grid lower [1,2]. Literature [2] proposed a three-level APF sliding model control, which is a traditional nonlinear control method that can stably control the harmonic content of the power grid, but the calculation is large, and the control has the problem of delay. MPC opinions are applied to many areas of power electronics, such as two-level converters, multi-level converters, and SVG.

The FCS-MPC of the three-level APF studied in this paper has a clear physical meaning and is easy to understand. It is improved from the traditional MPC to the field of converter control. The basic principle is to establish a discrete mathematical model of the control object, calculate the predicted current value according to different switch vector outputs, establish a value function, find the optimal switch combination under the minimum value function, and apply it to the APF. The APF DC side capacitor voltage adopts PI control, and the weight coefficient control is added to the three-level two capacitor voltage balance.

\section{The structure and mathematical model of three-level APF}

\subsection{Topology}

The main circuit structure diagram of the three-phase three-wire APF is shown in Fig.1. $e_{a}, e_{b}, e_{c}$ are the grid voltages, $i_{c a}, i_{c b}$ and $i_{c c}$ are the output compensation currents of the APF, $i_{L a}, i_{L b}$, and $i_{L c}$ are the load currents, and $L$ and $R$ are filter inductance and equivalent resistance, $C_{1}$ and $C_{2}$ are DC side balancing capacitors, and their capacitance values are equal.

\footnotetext{
$\overline{\text { *zhuruijin@xza.edu.cn }}$
} 


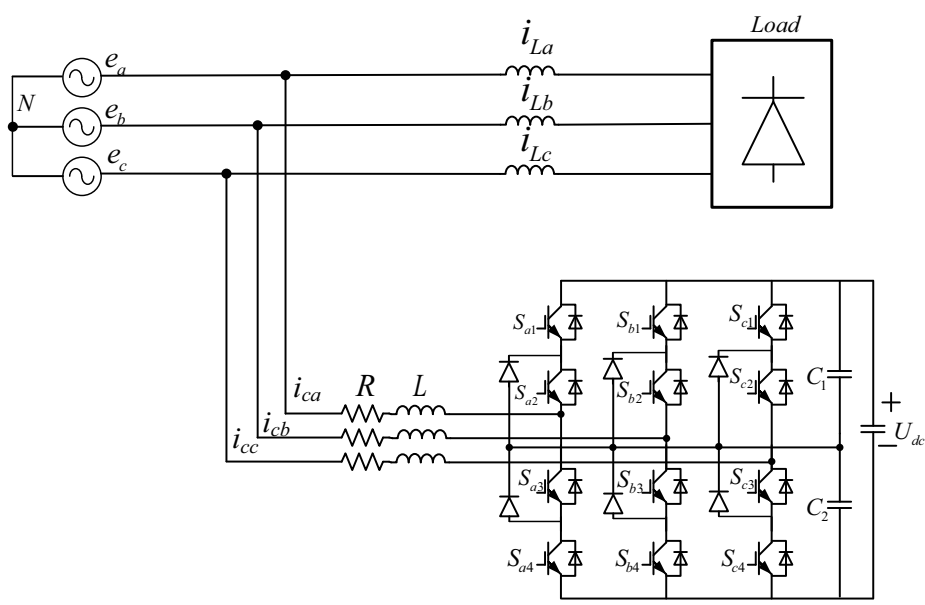

Fig.1 Structure diagram of three-level APF

For the three-level APF, each bridge arm has 4 points of IGBT, and there are 27 different switching states in total. From this, it can be defined that the formula of the switching function is equation (1).

$S_{x}= \begin{cases}P & S_{x 1}=S_{x 2}=1, S_{x 3}=S_{x 4}=0 ; \\ 0 & S_{x 1}=S_{x 4}=0, S_{x 2}=S_{x 3}=1 ; \\ N & S_{x 1}=S_{x 2}=0, S_{x 3}=S_{x 4}=1 ;\end{cases}$
The 27 switching states of the three-level APF can generate 19 different voltage vectors, including three sets of zero vectors and 12 sets of redundant vectors. These 27 switching states and vectors can be expressed as Fig. 2

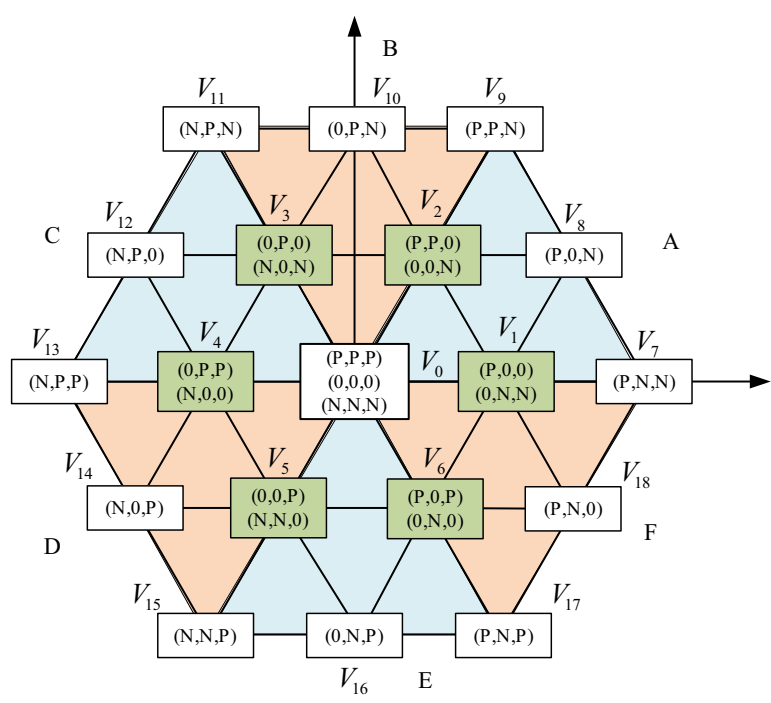

Fig.2 27 sets of switches vector

\subsection{Mathematical model of three-level APF}

According to the structure diagram of Fig. 1, the mathematical model of $a, b$ and $c$ three-phase can be obtained as equation (2), where $u_{c a}$ is the output voltage value of $\mathrm{APF}$.

$$
\left\{\begin{array}{l}
L \frac{\mathrm{d} i_{a}}{\mathrm{~d} t}=R i_{a}+e_{a}+u_{c a} \\
L \frac{\mathrm{d} i_{b}}{\mathrm{~d} t}=R i_{b}+e_{b}+u_{c b} \\
L \frac{\mathrm{d} i_{c}}{\mathrm{~d} t}=R i_{c}+e_{c}+u_{c c}
\end{array}\right.
$$

Since in the three-phase coordinate system, the current output tracking reference value will have cumulative errors, the mathematical model is established in the $\alpha-\beta$ coordinate system.

$$
\left\{\begin{array}{l}
L \frac{\mathrm{d} i_{\alpha}}{\mathrm{d} t}=u_{c \alpha}-e_{\alpha}-R i_{\alpha} \\
L \frac{\mathrm{d} i_{\beta}}{\mathrm{d} t}=u_{c \beta}-e_{\beta}-R i_{\beta}
\end{array}\right.
$$

The dynamic equation of DC side capacitor $\mathrm{C} 1, \mathrm{C} 2$ is equation (4)

$$
\begin{aligned}
\frac{\mathrm{d} u_{c 1}}{\mathrm{~d} t} & =\frac{1}{C} i_{c 1} \\
\frac{\mathrm{d} u_{c 2}}{\mathrm{~d} t} & =\frac{1}{C} i_{c 2}
\end{aligned}
$$




\section{3 finite control set model predictive control of there-level APF}

\subsection{FCS-MPC principle of current}

In the $\alpha-\beta$ coordinate system, there are 27 voltage vectors. The schematic diagram of predictive control is shown in Figure 3. At each sampling time, all switching states are cycled in turn, and the detected harmonic current value is taken as the reference value, and the variance is calculated with the predicted value. At the same time, the term of balancing capacitor voltage is added to distribute the weight factor, so as to synthesize all the reference tracking values, select the switch state with the minimum value function, output to APF, and cycle in the future. This way can track the harmonic current in real time without pulse width modulation (PWM).

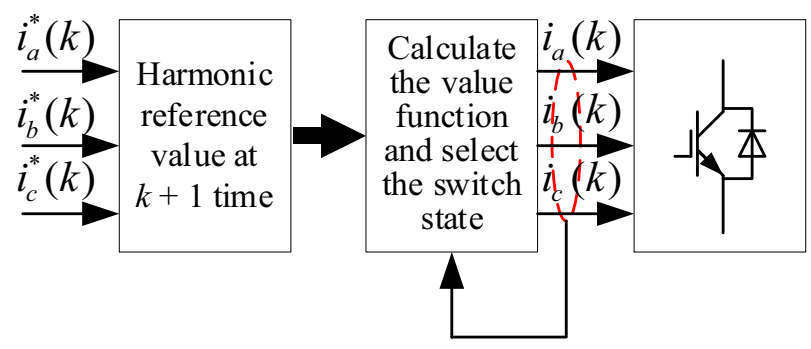

Fig.3 Diagram of FCS-MPC principle

\subsection{FCS-MPC of three-level APF}

After discretizing the difference of equations (3) and (4), equations (5) and (6) are obtained

$$
\left\{\begin{array}{c}
i_{\alpha}(k+1)=\frac{T_{s}}{L} u_{c \alpha}(k)-\frac{T_{s}}{L} e_{\alpha}(k)+\left(1-\frac{R T_{s}}{L}\right) i_{\alpha}(k) \\
i_{\beta}(k+1)=\frac{T_{s}}{L} u_{c \beta}(k)-\frac{T_{s}}{L} e_{\beta}(k)+\left(1-\frac{R T_{s}}{L}\right) i_{\beta}(k) \\
u_{c 1}(k+1)=\frac{1}{C} i_{c 1}(k) T_{s}+u_{c 1}(k) \\
u_{c 2}(k+1)=\frac{1}{C} i_{c 2}(k) T_{s}+u_{c 2}(k)
\end{array}\right.
$$

The $i_{c 1}(k)$ and $i_{c 2}(k)$ in equation (6) are the current values determined by the switch and the output current together, which are defined by equation (7).

$$
\begin{aligned}
& i_{c 1}(k)=i_{d c}(k)-G_{1 a} i_{a}(k)-G_{1 b} i_{b}(k)-G_{1 c} i_{c}(k) \\
& i_{c 2}(k)=i_{d c}(k)+G_{2 a} i_{a}(k)+G_{2 b} i_{b}(k)+G_{2 c} i_{c}(k)
\end{aligned}
$$

$G_{1 x}, G_{2 x}$ are determined by the current switch state, and their value is represented by equation $(8), i_{d c}(k)$ is the current on the DC side at the current moment.

$$
\begin{aligned}
& G_{1 x}=\left\{\begin{array}{l}
1, S_{x}=P \\
0, \text { others }
\end{array},\right. \\
& G_{2 x}=\left\{\begin{array}{l}
1, S_{x}=N \\
0, \text { others }
\end{array},\right.
\end{aligned}
$$

Since the reference harmonic current is at the current moment, the second-order LaGrange interpolation prediction is performed on it

$$
i^{*}(k+1)=4 i^{*}(k)-6 i^{*}(k-1)+4 i^{*}(k-2)-i^{*}(k-3)
$$

According to the above prediction equation, the cost function of equation (10) can be established

$$
J=\lambda_{1}\left\|i_{\alpha}^{*}(k+1)-i_{\alpha}(k+1)\right\|+\lambda_{2}\left\|i_{\beta}^{*}(k+1)-i_{\beta}(k+1)\right\|+\lambda_{3}\left\|u_{c 1}(k+1)-u_{c 2}(k+1)\right\|
$$

\section{Fixed Frequency FCS-MPC}

A new control strategy is proposed to solve the problems of unstable switching frequency and high sampling frequency in FCS-MPC of three-level APF. The optimal value of each voltage vector contained in the large area is determined by optimizing the value of each voltage sequence. Taking area, a as an example, the selection of switch vector is studied.

There are 10 groups of switch sequences in zone A. four groups of switch sequences decrease the neutral voltage and six groups increase the neutral voltage. There are a group of switching sequences that reduce the neutral point voltage and two groups of switch sequences that increase the neutral point voltage in the zone 1 and 2 . In zone 3 and zone 4, there are a group of switching sequences that affect the rise and fall of neutral point voltage. Taking area 1 and area 3 of area a as examples, the influence of switching sequence on neutral point voltage is illustrated, which is similar to other cells. In order to better explain the influence of three groups of switching sequence in zone 1 and zone 2 on neutral point voltage, the traditional space vector partition method is improved, that is, 1 is divided into 1.1 area and 12 area, and cell 2 is divided into 2.1 area and 2.2 area, as shown in Fig. 4. 


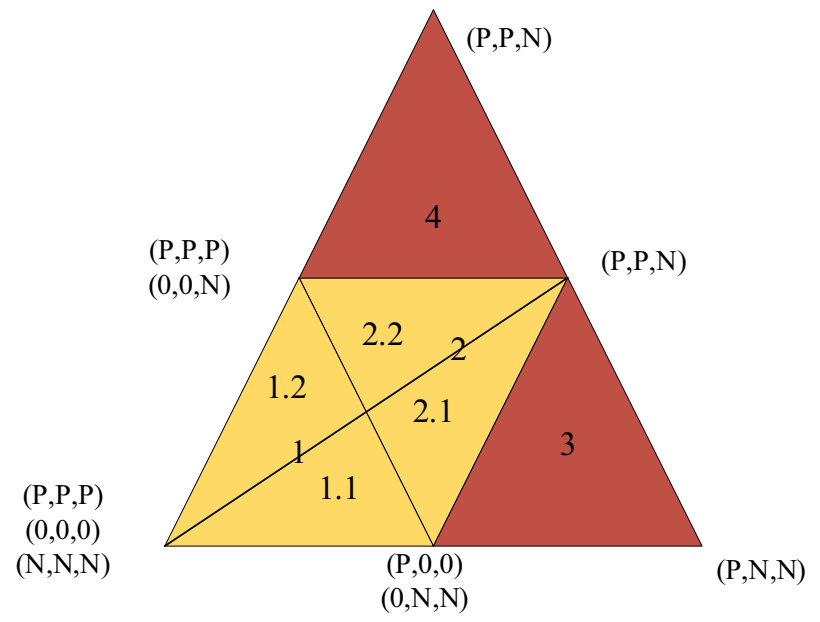

Fig.4 Division of area A

The reference voltage is located in zone 1 of area A, and the two groups of positive and negative small vectors $\mathrm{pp} 0 / 00 \mathrm{n}$ and $\mathrm{p} 00 / \mathrm{Onn}$ affect the neutral point voltage balance. The five-section switch sequence p00-000-00n000-p00 can reduce the neutral point voltage. Both small vector $\mathrm{p} 00$ and $00 \mathrm{n}$ can reduce the neutral point voltage, and the zero vector 000 has no effect on the neutral point voltage. Therefore, the five-segment switching sequence poo-p00-000-00n-000-p00 can reduce the neutral point voltage of three-level APF whether in 1.1 or 1.2 area. After determining the switch state to be output, the corresponding three voltage vectors can be determined. At this time, the duty cycle of the output vector in the rotating coordinate system can be calculated according to the reference vector.

$$
\begin{aligned}
& v_{r g}=d_{1} s_{1 g}+d_{2} s_{2 g}+d_{3} s_{3 g} \\
& v_{r h}=d_{1} s_{1 h}+d_{2} s_{2 h}+d_{3} s_{3 h} \\
& d_{1}+d_{2}+d_{3}=1
\end{aligned}
$$

By solving it, we can get the following results

$$
\left\{\begin{array}{c}
d_{1}=\frac{\left(v_{r g}-v_{3 g}\right)\left(v_{2 h}-v_{3 h}\right)-\left(v_{r h}-v_{3 h}\right)\left(v_{2 g}-v_{3 g}\right)}{\left(v_{1 g}-v_{3 g}\right)\left(v_{2 h}-v_{3 h}\right)-\left(v_{1 h}-v_{3 h}\right)\left(v_{2 g}-v_{3 g}\right)} \\
d_{2}=\frac{\left(v_{r g}-v_{3 g}\right)\left(v_{1 h}-v_{3 h}\right)-\left(v_{r h}-v_{3 h}\right)\left(v_{1 g}-v_{3 g}\right)}{\left(v_{2 g}-v_{3 g}\right)\left(v_{1 h}-v_{3 h}\right)-\left(v_{2 h}-v_{3 h}\right)\left(v_{1 g}-v_{3 g}\right)} \\
d_{3}=1-d_{1}-d_{2}
\end{array}\right.
$$

\section{Simulation Analysis}

In order to verify the effectiveness of the FCS-MPC control strategy for the three-level APF, a simulation model was built in MATLAB/Simulink, and the parameters are shown in Table 1. The harmonic source is an uncontrollable diode rectifier bridge, and the load fluctuates at 0.3 seconds. The reference current is the harmonics detected by the $i p$-iq method. Take $\lambda_{1}=\lambda_{2}=0.45$ and $\lambda_{3}=0.1$.
Table.1 Simulation model parameters

\begin{tabular}{cc}
\hline Parameters & Value \\
\hline Grid Voltage & $\mathbf{3 8 0 V}$ \\
DC Voltage & $\mathbf{8 0 0 V}$ \\
Capacitor $C_{1}, C_{2}$ & $\mathbf{3 0 0 0 \mu \mathbf { F }}$ \\
Inductor & $\mathbf{4 m H}$ \\
Resistor & $\mathbf{0 . 0 1 \Omega}$ \\
Load Resistor & $\mathbf{1 0 \Omega}$ \\
\hline
\end{tabular}

Settingf the above parameters of the simulation model, and running the simulation in Simulink, you can get the simulation diagrams in Fig. 5, which can reflect the dynamic performance and stability of the algorithm. 


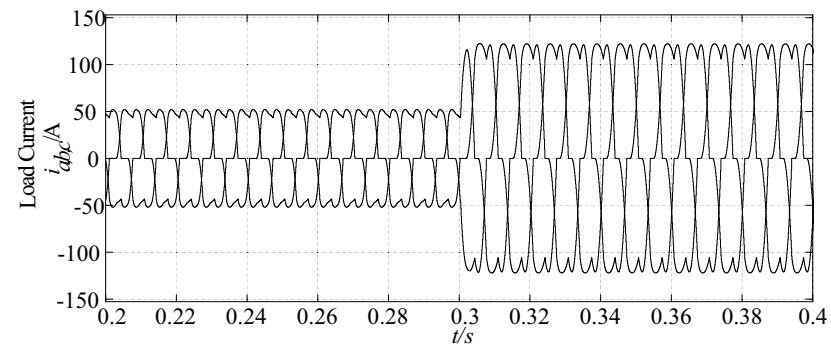

(a)

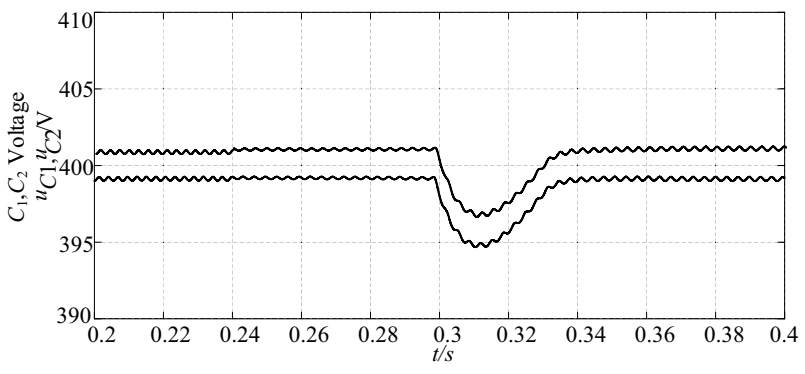

(b)

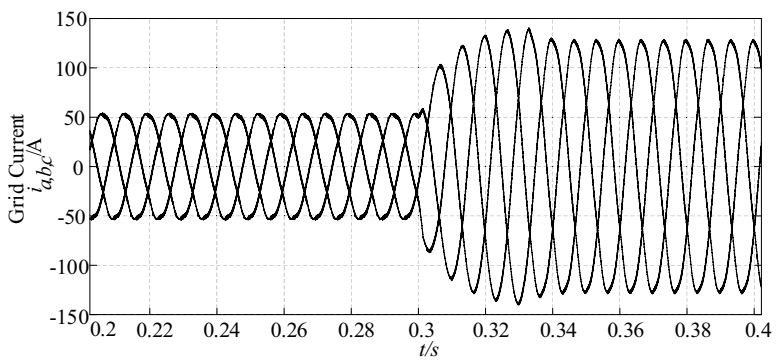

(c)

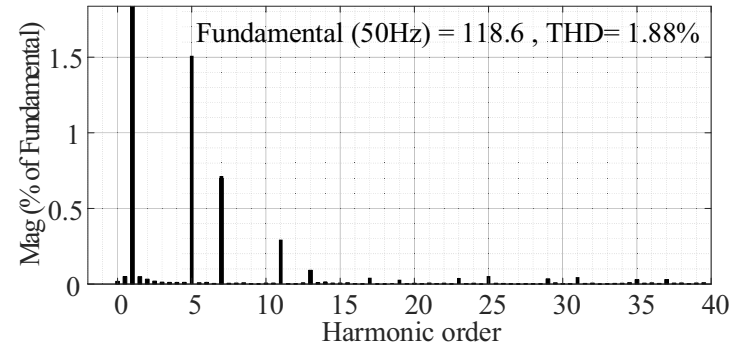

(d)

Fig.5 (a) is the three-phase load current; (b) is the voltage value of capacitors $C_{1}$ and $C_{2}$; (c) is the grid current after compensation;(d) is the total harmonic distortion rate at 2.35 seconds.

(a) In order to detect the three-phase harmonic load current, it can be seen from (a) that the harmonics of the power grid are mainly of the 3,5 and 7 harmonics, and the harmonic content is relatively high, which is detected by $i p-i q$ method, It can be seen from (b) that the capacitor voltage balance performance is good, and the expected value can be achieved quickly after the load changes. (c) In order to use FCS-MPC algorithm to control the APF compensation result, the current waveform is close to sine wave, and has fast dynamic performance. When the load changes, it can track the compensation harmonic well, and carry out FFT analysis on it. The THD of (d) is $2.07 \%$, which meets the harmonic requirements of power grid.

\section{Conclusion}

(a) Because of its structure, multi APF has more output voltage vector, better compensation effect and lower harmonic content. Aiming at the high sampling frequency and unfixed switching frequency of MPC algorithm, FCSMPC control algorithm is proposed. The output of the strategy is a group of vector sequences. The controlled object is determined as 10 groups of vector sequences, and the optimal vector sequence is determined by rolling optimization, It solves the problem of neutral point voltage offset of three-level ANPC inverter, and overcomes the disadvantage of traditional MPC algorithm that the switching frequency is not fixed. Through simulation, it has good performance, and the grid current 
keeps a low harmonic content, which proves the effectiveness and feasibility of the method.

\section{Acknowledgement}

Natural Science Foundation of Tibet Autonomous Region (XZ2019ZRG-69)

\section{References}

1. Vazquez S, Aguilera R, Acuna P, et al. Model Predictive Control for Single-Phase NPC Converters Based on Optimal Switching Sequences[J]. IEEE Transactions onIndustrial Electronics, 2016, 63(12):7533-7541.

2. Changliang Xia, Tao Liu, Tingna Shi, Zhanfeng Song. A Simplified Finite-Control-Set ModelPredictive Control for Power Converters [J]. IEEE Journals\&Magazines. 2014, vo1.10, no.2, pp. 9911002.

3. Vodyakho and C. C. Mi, "Three-Level InverterBased Shunt Active Power Filter in Three-Phase Three-Wire and Four-Wire Systems," in IEEE Transactions on Power Electronics, vol. 24, no. 5, pp. 1350-1363, May 2009, doi: 10.1109/TPEL.2009.2016663.

4. S. Sezen, A. Aktas, M. Ucar and E. Ozdemir, "A three-phase three-level NPC inverter based gridconnected photovoltaic system with active power filtering," 2014 16th International Power Electronics and Motion Control Conference and Exposition, Antalya, 2014, pp. 1331-1335, doi: 10.1109/EPEPEMC.2014.6980697.

5. B. Singh, K. Al-Haddad, and A. Chandra, "Active power filter with sliding mode control," in IEE Proceedings - Generation, Transmission and Distribution, vol. 144, no. 6, pp. 564-568, Nov. 1997, doi: 10.1049/ip-gtd:19971431.

6. A. Fahmy, M. S. Hamad, A. K. Abdelsalam and A. Lotfy, "Power quality improvement in three-phase four-wire system using a shunt APF with predictive current control," IECON 2012 - 38th Annual Conference on IEEE Industrial Electronics Society, Montreal, QC, 2012, pp. 668-673, doi: 10.1109/IECON.2012.6388748. 